Artí́culo Original

\title{
Prevalencia de anomalías dentarias en pacientes con fisura labio alveolo palatina atendidos en el Instituto Especializado de Salud del Niño. Lima, Perú
}

Prevalence of dental anomalies in patients with cleft lip alveolus and palate treated at Institute Specialized on Children's Health. Lima, Perú

\section{Resumen}

Se estudió las anomalías en número de las piezas dentarias en pacientes con fisura labio alveolo palatina (FLAP) utilizando radiografías panorámicas e historias clínicas de 129 niños (79 niños y 50 niñas) de 6 a 12 años, del Instituto Especializado de Salud del Niño, Lima, Perú, correspondiente a los años 2005 a 2008. La prevalencia de agenesia dentaria fue de $86.8 \%$ y de dientes supernumerarios de $49.6 \%$ de la población total, mientras que $43.4 \%$ presentó ambas anomalías a la vez. 78 pacientes mostraron FLAP unilateral (60.5\%); y 51, FLAP bilateral (39.5\%). Con respecto a la distribución de agenesia dentaria según género se apreció que 68 casos $(60,7 \%)$ fueron del género masculino y 44 del género femenino $(39,3 \%)$; mientras que en los dientes supernumerarios 42 casos $(65.6 \%)$ de género masculino y 22 casos (34.4\%) del género femenino. Comparando la frecuencia de agenesia y dientes supernumerarios con el tipo de fisura, el grupo más afectado fue el de FLAP bilateral con 48 casos (42.9\%) y 27 (42.2\%) respectivamente, en cuanto al grupo de FLAP unilateral el del lado izquierdo presentó mayor afectación en ambas anomalías dentarias. La agenesia dentaria se presentó mayormente en el lado opuesto a la fisura (96.4\%) mientras que los dientes supernumerarios se observan generalmente en el lado de la fisura (92.2\%). Según la cantidad de piezas afectadas 41 niños presentaron una agenesia $(36,6 \%)$ y 34 niños con dos agenesias (30,4\%), siendo las piezas más afectadas el incisivo lateral (92.9\%), segunda premolar (38.4\%). En el caso de dientes supernumerarios, 39 casos $(60.9 \%)$ presentaron sólo una pieza y 23 casos (35.9\%) presentaron dos piezas supernumerarias; ubicados frecuentemente en el espacio entre el incisivo lateral y canino superior, presentándose por lo general no eruptivo (79.7\%). Los dientes supernumerarios heteromórficos fueron los más comunes en este estudio.

\section{Abstract}

The Prevalence and distribution of dental anomalies in patients with cleft lip alveolus and palate (CLAP) were studied using panoramic x-rays of 129 children (79 boys and 50 girls), 6 to 12 year old belonging the Institute specialized on Children's Health, Lima - Perú, during 2005 to 2008. Hypodontia prevalence of $86.8 \%$ and supernumerary teeth prevalence in the $49.6 \%$ was found in the population. Both anomalies were simultaneously found in $43.4 \%$. The studied population showed 78 patients with unilateral CLAP (60.5\%) and 51 with bilateral CLAP (39.5\%). According to the relationship between hypodontia distribution and gender, 68 cases $(60.7 \%)$ were boys and 44 girls $(39.3 \%)$; whereas in the supernumerary teeth, 42 cases $(65.6 \%)$ boys and 22 cases were girls $(34,4 \%)$. Comparing frequency of hypodontia and supernumerary teeth with the type of cleft, the most affected group was the bilateral CLAP group with 48 cases $(42.9 \%)$ and $27(42.2 \%)$ respectively, whereas the group of unilateral left CLAP was the one which were more affected in both dental anomalies. Hypodontia appeared mainly in the opposed side to the cleft $(96,4 \%)$ whereas supernumerary teeth are observed generally in the cleft side (92,2\%). 41 children shown hypodontia in one tooth $(36.6 \%)$ and 34 children showed only $2(30.4 \%)$, being more affected the maxillary lateral incisor $(92,9 \%)$ and maxillary second premolar (38.4\%). Most of the patients (60.9\%) had 1 supernumerary tooth and 23 patients $(35.9 \%)$ had 2 supernumerary teeth; placed frequently in between the maxillary lateral incisor and maxillary canine, appearing generally non erupted $(79.7 \%)$. Most of the supernumerary teeth had a non typical shape.

\section{INTRODUCCIÓN}

Las deformidades del labio, alveolo y paladar hendido son malformaciones congénitas producidas por la no unión de algunos procesos faciales y que se presentan comúnmente en pacientes de todo el mundo, encontrándose en muchas investigaciones realizadas respecto a esta anomalía un promedio de incidencia de 1 en 1000 casos por año ${ }^{1}$.

\section{Luis Alberto Mogollón Tello ${ }^{1}$ Olinda Huapaya Paricoto ${ }^{2}$}

\begin{tabular}{ll}
\hline & CD. Práctica privada. \\
2 & Dpto. Académico Médico Quirúrgico. Facultad \\
& Odontología. UNMSM. Lima, Perú.
\end{tabular}

Correspondencia:

CD. Luis Alberto Mogollón Tello

Dirección: Calle Los Fresnos D1-6 Urb. Residencial Monterrico La Molina. Lima, Perú

Teléfono: 4355260 / 997452671

E-mail: mogollon_tello@hotmail.com
Palabras clave: Agenesia dentaria. Fisura labio alveolo palatina. Dientes supernumerarios.
Keywords: Hypodontia. Cleft lip and palate. Supernumerary teeth.
Existen múltiples investigaciones realizadas respecto a estas anomalías donde se concluye que estas malformaciones se presentan debido a un sin número de factores que intervienen durante el desarrollo intrauterino; es así que en la 
actualidad se habla no sólo de un factor hereditario, sino también de factores ambientales como causantes de estas patologías. Se ha observado que las deformidades producidas en el labio y el paladar se originan en las primeras siete semanas de vida en el útero, cuando se forma el labio y la premaxila ${ }^{2}$.

Las hendiduras labio alveolo palatinas originan problemas en las estructuras óseas del maxilar superior presentando así una alteración en el perfil facial, además de muchas veces encontrar comunicaciones oronasales ocasionando problemas en la deglución, respiración y fonética del paciente.

Además, el desarrollo del proceso alveolar en la región del paladar de estos pacientes determina una serie de anomalías dentarias tanto en su número y forma, lo que constituye un factor etiológico de maloclusiones que se encuentran en la totalidad de pacientes fisurados ocasionando problemas funcionales y estéticos. De esta manera, el cirujano dentista debe considerar estos desórdenes para establecer un plan de tratamiento y buscar la armonía del arco dentario, como parte importante de un equipo multidisciplinario encargado del tratamiento integral de estos pacientes.

La ausencia congénita de determinados dientes es un trastorno común en nuestra población. Los dientes que más suelen faltar en la población normal son los terceros molares, los segundos premolares, los incisivos laterales superiores y centrales inferiores. La hipodoncia es más frecuente entre los asiáticos y los nativos americanos que entre los blancos ${ }^{3}$. En el Perú, actualmente se registra una frecuencia de $9.7 \%$ de la población estudiada que presenta esta anomalía dentaria ${ }^{4}$.

Aunque pueden aparecer en cualquiera de las denticiones, los dientes supernumerarios son más frecuentes en la dentición permanente. Su forma es variable, desde normal a cónica. Aunque se desconoce su etiología existe una tendencia familiar. La mayoría de los casos son poligénicos y representan mutaciones genéticas espontáneas iniciales ${ }^{3}$.

Existen varias teorías que tratan de explicar el origen de los dientes supernumerarios como la hiperactividad de la lámina dental donde ocurre una proliferación celular, factores hereditarios, división completa del germen dentario y la influencia genética. La prevalencia en la población general es de $0.3 \%$ al $3.8 \%$ aunque hay reportes que señalan que hay una prevalencia de 8 a $9.1 \%{ }^{5}$.

En el Perú se observó, en el año 2003, una frecuencia de $4.3 \%$ en una población de niños con dentición mixta y permanente temprana ${ }^{4}$.

En dentición permanente, estos dientes supernumerarios van a condicionar alteraciones eruptivas con apińamiento retenciones o desviaciones de los dientes vecinos. Igualmente pueden dar lugar a diastemas reabsorciones radiculares e incluso formaciones quísticas. Todo diente supernumerario debe ser extraído ${ }^{6}$.

La frecuencia de dientes supernumerarios en los niños hace imprescindible la valoración radiográfica hacia los 5 ó 6 años, antes de la erupción de los incisivos permanentes superiores ${ }^{7}$.

El presente estudio tuvo como propósito determinar las alteraciones en número como agenesia dentaria y dientes supernumerarios en pacientes con fisura labio alveolo palatina con la ayuda del análisis radiográfico y la historia clínica. La alta prevalencia de estas anomalías en número de piezas dentarias encontradas en este estudio nos lleva a fijar importancia en el diagnóstico temprano y tratamiento oportuno en estos pacientes. La importancia de esta investigación es la de identificar cuál es la prevalencia de dientes ausentes y supernumerarios en los pacientes con fisura labio alveolo palatina, en una población peruana y determinar cuáles son los dientes presentes y ausentes para el diagnóstico temprano y así lograr la armonía en las arcadas maxilares.

\section{MATERIAL Y MÉTODOS}

La muestra estuvo constituida de 129 historias clínicas y radiografías panorámicas pertenecientes a pacientes con fisura labio alveolo palatina tomadas entre los 6 y 12 años, y que fueron atendidos en la Unidoad de Fisurados del Servicio de Ortodoncia y Ortopedia Maxilar del Instituto Especializado de Salud del Niño entre enero del 2005 a junio del 2008. Dicho número de radiografías e historias clínicas constituyó el número total de la población a estudiar.

Dichos pacientes tuvieron como requisito no presentar síndromes asociados y haber sido intervenidos quirúrgicamente en el IESN.

La muestra seleccionada se determinó de manera no aleatoria con la finalidad de estudiar solo a los pacientes de 6 a 12 años con fisura labio alveolo palatina, trabajándose con un total de 129 historias clínicas con sus respectivas radiografías panorámicas, radiografías cefalométricas y fotografías extraorales e intraorales. Se tomó en cuenta como referencia la edad del niño en el momento en que se tomó la radiografía panorámica.

\section{Procedimientos y técnicas}

Los datos obtenidos en cada caso fueron registrados en una ficha que se elaboró para este estudio, donde se registró el nombre, edad y sexo del paciente; el tipo de fisura; así como el tipo de alteración en el número de piezas dentaria que presentaba, la ubicación, tipo de pieza dentaria afectada y la cantidad de alteraciones presentes.

La evaluación se realizó con la ayuda de negatoscopio y lupa para una observación minuciosa, enfatizándose en los siguientes aspectos:

Número de dientes en la radiografía panorámica.

- Se contaron piezas ausentes y la presencia de dientes supernumerarios.

Basados en la clasificación de Veau de fisuras labio palatinas y siguiendo el criterio propuesto para esta investigación por la Unidad de Fisurados del Servicio de Ortodoncia y Ortopedia maxilar del Instituto de Salud del Niño se utilizó una clasificación para los pacientes con fisura labio alveolo palatina que es la siguiente:

- Fisura labio alveolo palatina.

- Fisura labio alveolo palatina izquierda.

- Fisura labio alveolo palatina derecho.

Luego de obtenidos los datos, fueron procesados para aislar la información y los hallazgos importantes. Se verificó que los datos obtenidos sean exactos $y$ completos.

Una vez recolectados los datos y registrados en la base de datos Excel, se trabajó con el programa SPSS computarizado para la obtención de los datos estadísticos, elaboración de tablas y gráficos.

Los resultados del estudio, en cuanto a la prevalencia y distribución de agenesia dentaria y dientes supernumerarios, se evaluaron en tablas de distribución de frecuencia y tablas de contingencia. Además se elaboró gráficos de prevalencia y distribución porcentual para representar las tablas.

\section{RESULTADOS}

De los 129 niños con fisura labio alveolo palatina, 36 niños presentaron fisura labio alveolo palatina unilateral en el lado derecho (27.9\%), 42 niños 
presentaron la fisura unilateral en el lado izquierdo (32.6\%) y 78 niños presentaron fisura labio alveolo palatina bilateral (39.5\%).

Tabla 1. Distribución según tipo de anomaedad con fisura labio alveolo palatina. 2005 -2008 .

\begin{tabular}{lcc}
\hline Anomalía dentaria & No & $\%$ \\
\hline $\begin{array}{l}\text { Agenesia dentaria } \\
\text { Diente }\end{array}$ & 112 & 86.8 \\
$\begin{array}{l}\text { Supernumerario } \\
\text { Ambos }\end{array}$ & 64 & 49.6 \\
\hline
\end{tabular}

Se observó un total de 112 niños con agenesia dentaria que representa el $86.8 \%$ de la población y 64 niños que presentan dientes supernumerarios que constituyen un $49.6 \%$ de la población total.

De los 112 niños afectados con agenesia, 104 presentaron agenesia del incisivo lateral (92.9\%), seguido por 43 niños que presentaron agenesia del segundo premolar $(38.4 \%)$, agenesia del incisivo central en $19(17.0 \%)$, primera premolar en 4 (3.6\%), canino y primer molar en 3 nińos $(2.7 \%)$ cada uno, y segunda molar en 1 niño $(0.9 \%)$.

Para determinar la ubicación de la agenesia dentaria en relación al lado de la fisura, se obtuvo que de 112 niños, 108 (96.4\%) presentaron la agenesia en el lado opuesto de la fisura, 43 (38.4\%) presentaron la agenesia en el lado de la fisura y $39(34.8 \%)$ encontraron la agenesia en ambos lados.

Evaluando la agenesia dentaria, según el tipo de fisura labio, resultó que de 112 niños el grupo más afectado fue el de fisura labio alveolo palatina bilateral con 48 niños (42.9\%), 36 fueron del grupo de FLAP unilateral izquierda (32.1\%), mientras que 28 fueron FLAP unilateral derecha $(25.0 \%)$. lía dentaria en pacientes de 6 a 12 ańos de

En cuanto a la distribución del tipo de pieza dentaria supernumeraria que se encontró en los nińos con mayor frecuencia, se presentó la siguiente secuencia en orden decreciente. De los 64 niños que presentaron dientes supernumerarios, 52 de ellos presentaron dientes supernumerarios heteromórficos $(81.3 \%), 14$ niños presentó incisivo lateral de tipo eumórfico $(21.9 \%)$, 2 presentaron canino supernumerario eumórfico $(3.1 \%)$ y sólo 1 presento incisivo central supernumerario (1.6\%).

Con respecto a los dientes supernumerarios según el tipo de fisura; de 64 niños el grupo más afectado fue también el de FLAP bilateral con 27 (42.2\%), seguido por el de FLAP unilateral izquierda con 20 nińos (31.3\%) y el de FLAP unilateral derecha con 17 niños $(26.6 \%)$

En cuanto a la ubicación del diente supernumerario en relación al lado de la fisura, resultó que de 64 nińos, 59 $(92.2 \%)$ tuvieron el diente supernumerario en el lado de fisura, $9(14.1 \%)$ la tuvieron en el lado opuesto de la fisura mientras que $5(7.8 \%)$ presentaron el diente supernumerario en ambos lados.

\section{DISCUSIÓN}

En el presente estudio referente a las alteraciones en número de las piezas dentarias en pacientes de 6 a 12 años de edad con fisura labio alveolo palatina unilateral o bilateral atendidos en el ISN entre los ańos 2005-2008, se encontró una frecuencia de agenesia dentaria de $86.8 \%$ así como una frecuencia de $49.6 \%$ para dientes supernumerarios. Dichas frecuencias son estadísticamente más alta al $9.7 \%$ para agenesias y al $4.3 \%$ para dientes supernumerarios, reportadas para una población peruana de no fisurados ${ }^{4}$.

Con respecto a la frecuencia de agenesia dentaria, comparando con estudios previos, se observa una frecuencia más

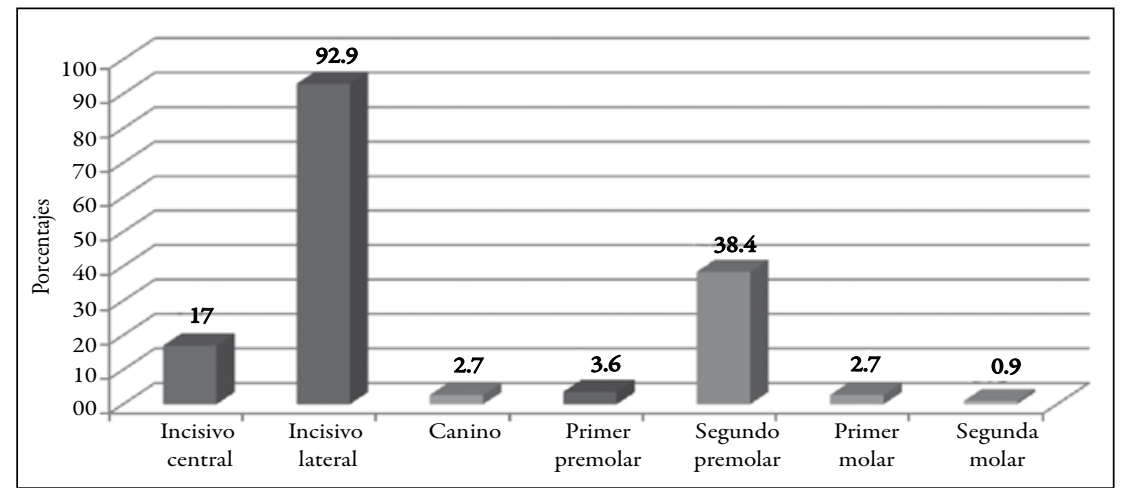

Gráfico $\mathbf{N}^{\circ}{ }^{\circ}$. Dsitribución del tipo de diente afectado por agenesia. alta a los obtenidos por otros autores con $77 \%{ }^{8}, 67.6 \%{ }^{9}$ y $72.4 \%{ }^{10}$. En cuanto a la frecuencia de dientes supernumerarios que se obtuvo en el presente estudio (49.6\%), se encontró elevada con respecto a las encontradas por Slayton $(2003)^{11}$ de $27 \%$ y Alas $(2007)^{10}$ con $27.6 \%$, y notoriamente distinta al rango de frecuencia en estudios previos entre $2.7 \%$ y $7.3 \%$ hallados por Tsai $(1998)^{12}$, Kim (2006) ${ }^{13}$, Ribeiro $(2003)^{14}$ y Heliovaara $(2004)^{15}$. Esta discrepancia puede entenderse por el criterio usado para este estudio, donde se consideró a los dientes malformados como supernumerarios y no como incisivos laterales.

El tipo de fisura con mayor frecuencia fue de tipo unilateral representando el $60.5 \%$ del total de la muestra siendo el lado izquierdo el más común, en concordancia con los estudios de Shapira $(1999)^{16}$ y Lekkas $(2000)^{17}$;

Del grupo de pacientes que presentaban agenesia dentaria, los de FLAP bilateral fueron más frecuentes $42.9 \%$. Esta frecuencia coincide con lo encontrado por Shapira $(2000)^{8}$ y confirma que mientras más severa la fisura mayor es la alteración en número de dientes.

La frecuencia de agenesia dentaria en el lado de fisura $(38.4 \%)$ y en el lado opuesto $(96,4 \%)$ están en contraste con las encontradas por Baek y Kim $(2007)^{13}$ y Shapira $(2000)^{8}$, autores que encuentran mayor frecuencia en el lado de fisura. Esto puede ser explicado debido al criterio que se tomó en cuenta en este estudio, al considerar la línea de fisura como centro de la división de lados.

Con respecto a la cantidad de piezas afectadas con agenesia en cada paciente; $36.6 \%$ presentó sólo una pieza congénitamente ausente, $30.4 \%$ presentó dos y el $19.6 \%$ presentó tres dientes con agenesia; resultados similares a los mostrados por Heliovaara (2004) ${ }^{15}$, sin embargo en nuestro estudio no se consideró las ausencias congénitas en la mandíbula.

Evaluando la agenesia dentaria con relación a la morfología dental, del total de pacientes con agenesia, $92.9 \%$ tuvieron agenesia del incisivo lateral; $38.4 \%$, del segundo premolar; y $17.0 \%$, del incisivo central. Este hallazgo es comparable a lo descrito por la mayoría de estudios previos pero en conflicto con los resultados de Heliovaara (2004) ${ }^{15}$ donde se presenta al segundo premolar inferior con mayor frecuencia, seguido por el incisivo lateral superior y segundo premolar superior. Esta discrepancia puede 
ser debido a que este autor estudio a pacientes con un determinado tipo de fisura leve y cuya frecuencia de agenesia dentaria estaría más en correspondencia con la frecuencia de agenesias en la población normal.

En este estudio, $81.3 \%$ del total de pacientes con presencia de dientes supernumerarios fueron de tipo heteromórfico, mientras que $21.9 \%$ de pacientes presentaron dientes supernumerarios eumórficos. Si consideramos al mesioden como un diente heteromórfico, estos resultados concuerdan con los encontrados por Alas $(2007)^{10}$ y Tsai y col $(1998)^{12}$.

Al término de la investigación se llegó a las siguientes conclusiones:

Este estudio reveló una mayor frecuencia de fisura labio alveolo palatina unilateral que bilateral, y a su vez, mayor frecuencia en el lado izquierdo. Sin embargo, el grupo que resultó más afectado con agenesias dentarias y dientes supernumerarios es el de fisura labio alveolo palatina bilateral. Los nińos afectados con agenesia dentaria presentaron en su mayoría la ausencia de 1 ó 2 dientes, así como los nińos afectados con dientes supernumerarios presentaron en su mayoría 1 solo diente en exceso.

\section{REFERENCIAS BIBLIOGRÁFICAS}

1. Ortiz B. prevalencia de Labio y/o Paladar Fisurado en neonatos y factores de riesgo. Instituto Especializado Materno Perinatal (20012002). [Tesis para optar el título de Cirujano Dentista] Lima: UPCH; 2003.

2. Arcaya G. Frecuencia de fisuras labiales, palatinas y labio palatinas en el Hospital Nacional Cayetano Heredia y en pacientes de la Clínica Estomatológica Central durante los años 1994 a 1999. [Tesis para obtener el título de Cirujano Dentista] Lima: UPCH; 2001.

3. Goaz P. Radiología Oral. Principios e Interpretación. $3^{a}$ ed. Madrid-España: Mosby/Doyma Libros; 1995.

4. Alcántara M. Prevalencia y Distribución de Agenesias Dentarias y Dientes Supernumerarios en Pacientes de 7 a 18 ańos de edad atendidos en el Centro Médico Naval. Enero 2003 - Julio 2004. [Tesis para obtener el título de Cirujano dentista] Lima: UNMSM; 2003.

5. Ponce y col. Dientes supernumerarios en una población infantil del Distrito Federal. Estudio clínico radiográfico. Revista de la Asociación Dental Mexicana 2004; 61(5).

6. Abramowics y col. Demographic and prenatal factors of patients with cleft lip and palate. J American Dental Association. 2003 Oct; 134:1371-1376.

7. Labio y paladar hendido. Artículo disponible en URL: http://www. mexdoctors.com/seamanduras/docs/ LabioLeporino.pdf

8. Shapira Y, Lubit E, Kuftinec M. Hypodontia in children with various types of clefts. The Angle Orthodontist. 2000 Feb; 1(70).

9. Aizenbud D. y col. Congenitally missing Teeth in the Israeli Cleft Population. The Cleft Palate-Craniofacial Journal. 2005; 42(3):314317.

10. Alas y col. Incidencia de dientes ausentes y supernumerarios en pacientes con labio y paladar hendido. Ortodoncia. Revista Latinoamericana de Ortodoncia y Ortopedia. 2007; (6). Disponible en: www.ortodoncia.ws/publicaciones/
11. Slayton y col. Genetic Association Studies of Cleft Lip and/or Palate With Hypodontia Outside the Cleft Region. The Cleft Palate-Craniofacial Journal. 2003; 40(3):274279.

12. Tsai TP, Huang CS, Huang CC, See LC. Distribution patterns of primary and permanent dentition in children with unilateral complete cleft lip and palate. The Cleft Palate-Craniofacial Journal. 1998; 35:154-60.

13. Baek, S. y Kim, N. Congenitally missing permanent teeth in korea unilateral cleft lip and alveolus and unilateral cleft lip and palate patients. Angle Orthodontist. 2007; 7(1):88-93.

14. Ribeiro L. y col. Dental anomalies of the permanent lateral incisors and prevalence of hypodontia outside the cleft area in complete unilateral cleft lip and palate. The Cleft Palate-Craniofacial Journal 2003; 40(2):172-175.

15. Heliovaara A, Ranta R, Rautio J. Dental abnormalities in permanent dentition in children with submucous cleft palate. Acta Odontológica Escandinava. 2004 junio; 62(3):129-31

16. Shapira Y. Congenitally missing second premolar in cleft lip and cleft palate. American Journal of orthodontics and Dentofacial Orthopedics. 1999; 115:396-400.

17. Lekkas, C. The adult unoperated cleft patient: Absence of maxillary teeth outside the cleft area. The Cleft Palate-Craniofacial Journal. 2000; 37(1):17-20.

Recibido: 17-11-08

Aceptado para publicación: 12-12-08 